\title{
Un faciès forestier relictuel : les chênaies montagnardes, des Vosges lorraines
}

In search of vanished oak forests in the Vosges mountains

Ein Reliktwaldfazies: Die Gebirgseichenwiilder der lothringischen Vogesen

\section{Xavier Rochel}

\section{(2) OpenEdition}

\section{Journals}

Édition électronique

URL : http://journals.openedition.org/rge/1532

DOI : $10.4000 /$ rge.1532

ISSN : 2108-6478

\section{Éditeur}

Association des géographes de l'Est

Édition imprimée

Date de publication : 1 septembre 2007

ISSN : 0035-3213

\section{Référence électronique}

Xavier Rochel, "Un faciès forestier relictuel : les chênaies montagnardes, des Vosges lorraines », Revue Géographique de l'Est [En ligne], vol. 47 / 4 | 2007, mis en ligne le 16 janvier 2012, consulté le 08 septembre 2020. URL : http://journals.openedition.org/rge/1532 ; DOI : https://doi.org/10.4000/rge. 1532

Ce document a été généré automatiquement le 8 septembre 2020.

Tous droits réservés 


\title{
Un faciès forestier relictuel : les chênaies montagnardes, des Vosges lorraines
}

\author{
In search of vanished oak forests in the Vosges mountains \\ Ein Reliktwaldfazies: Die Gebirgseichenwiilder der lothringischen Vogesen
}

\author{
Xavier Rochel
}

\section{Introduction}

1 Si le sapin est bien l'arbre roi du massif vosgien, il n'occupe pas cette place depuis toujours. L'évolution de la végétation vosgienne s'est opérée avec lenteur, au rythme des oscillations climatiques des derniers millénaires, et s'est brusquement accélérée avec la mise en place d'une exploitation et d'une sylviculture intensives à partir du XVIe siècle. Même au cours des quatre derniers siècles, se sont succédées des périodes de brusques accélérations dans la transformation des forêts, et des périodes de stabilité relative.

Le travail en archives reste clairement un outil incontournable pour l'histoire de l'environnement à cette échelle de temps, de l'ordre de quatre à cinq siècles au maximum (Agnoletti, Anderson, 2000) et même les études entreprises à partir des archives du sol ne peuvent être déconnectées des textes qui en éclairent le sens (Bonhôte 1998). Dans le cas vosgien, l'étude de certaines archives forestières fait apparaître avec clarté les forts changements intervenus dans les derniers siècles et permet de reconstituer, avec des résultats forcément limités, la forêt vosgienne d'avant les grands bouleversements du XIXe siècle. Ce travail a été entrepris pour les hêtraiessapinières montagnardes (Rochel 2004a), les hêtraies-chênaies collinéennes (Rochel 2007), les hêtraies du piémont (Rochel 2008) ; il peut utilement compléter les travaux entrepris à partir des archives naturelles (Nölken 2005), et s'inscrit dans une suite de 
travaux de biogéographie historique initiée notamment par Gérard Houzard (1980), Jean- Jacques Dubois $(1986,1989)$ ou encore Jean-Pierre Husson.

3 Parmi les changements les plus spectaculaires qui ont affecté les forêts vosgiennes, figure le cas des chênaies montagnardes. La chênaie sessiliflore n'est aujourd'hui présente que de façon tout à fait marginale sur le versant lorrain des Vosges ${ }^{1}$, alors qu'elle est bien présente du côté alsacien. Elle n'occupe que quelques dizaines d'hectares, sous la forme de peuplements réduits², en versant sud, sur des pentes escarpées au sol maigre. Or, la chênaie est omniprésente dans les archives des XVIe, XVIIe et XVIIIe siècles et semble avoir occupé, jusqu'à une date assez récente, des surfaces tout à fait notables. La place importante occupée jadis par le chêne dans le massif est établie depuis longtemps (Dion 1985), mais avec peu de données. Il reste donc à préciser, à l'aide des corpus écrits laissés par les forestiers lorrains, la localisation, la composition, et le rôle ancien de ces peuplements de chêne autrefois désignés sous le terme « rapailles », et généralement enrésinés au cours des 150 à 200 dernières années.

4 Les archives disponibles ne sont pas nombreuses. Le récolement des corpus du XIXe siècle se révèle malheureusement décevant : ces archives ne sont vraiment abondantes qu'à partir des années 1840-1850 et à cette date, la plupart des peuplements en chênaie étaient déjà en cours d'enrésinement, en tout cas en ce qui concerne les forêts prospectées à ce jour. La caractérisation des chênaies montagnardes doit donc passer par les archives des forestiers ducaux du XVIIIe siècle, lesquelles peuvent être paradoxalement plus intéressantes, et mieux exploitables que celles de leurs homologues du siècle suivant. C'est donc à partir des archives de la maîtrise des Eaux et Forêts de Saint-Dié, essentiellement, qu'il est possible de reconstituer partiellement les " rapailles » feuillues des Vosges lorraines. On abordera donc successivement le problème des rapailles vosgiennes et l'enjeu qu'elles représentent, le problème des sources disponibles et de la démarche à adopter, les résultats de la recherche et les enseignements qu'il faut en tirer.

\section{Des peuplements relictuels?}

\section{A. Les chênaies sur le versant lorrain des Vosges}

5 Pour reprendre les termes du géographe Georges Savouret, le massif vosgien est en quelque sorte une " montagne-forêt » (Savouret 1985, p. 6), à l'image des montagnes hercyniennes en -wald de l'Europe médiane : Schwarzwald, Thüringerwald, Böhmerwald. Les surfaces boisées y sont considérables : le taux de boisement atteint 77 $\%$ dans les Vosges gréseuses (« Hautes Vosges gréseuses " selon la terminologie de l'I.F.N.), 70 \% dans les Hautes Vosges cristallines ; de plus, l'exploitation du bois et de ses sous-produits représente un pan considérable de l'économie vosgienne. Si cette forêt reste dominée par les résineux (dans la région forestière "Hautes Vosges gréseuses ", la part des conifères dans le volume sur pied atteint $84 \%)^{3}$, et si le sapin est incontestablement l'arbre-roi du massif, les feuillus ne sont pas absents. Dans l'étagement, s'observent d'abord la hêtraie et les hêtraieschênaies plus ou moins acidiphiles du pourtour du massif ; à l'étage montagnard, la hêtraie-sapinière a souvent été volontairement appauvrie en feuillus mais conserve parfois encore son caractère mixte ; à proximité des sommets, les hêtraies dites sommitales ou d'altitude, pour partie intégrées à l'étage subalpin, constituent encore des peuplements feuillus de 
surface importante. En outre, dans certaines stations caractérisées par des conditions topographiques et/ou édaphiques très particulières, les feuillus dominent presque complètement la végétation potentielle, au point d'exclure souvent les résineux : citons les érablaies d'éboulis, les aulnaies et aulnaies-saulaies ripicoles, mais aussi les chênaies acidophiles de versant Sud.

Sur le versant alsacien, la chênaie sessiliflore acidophile est assez largement présente, en particulier dans les collines sous-vosgiennes et en versant sud (plus rarement nord) de la partie aval des principales vallées, Fecht ou Bruche par exemple. Rattachée au Quercionrobori petraeae, la chênaie y est généralement traitée en taillis ou, surtout, en anciens taillis convertis en une futaie de qualité très médiocre. Mais sur le versant lorrain du massif, la chênaie reste une exception, au point d'être négligée par les publications généralistes sur la forêt lorraine. Marcel Jacamon (1983) ne la mentionne pas. Dans sa thèse (1985), Jean Dion ne lui accorde qu'une place très médiocre, à l'inverse du versant alsacien, et il n'apporte pas d'informations certaines et vérifiées.

7 Encadrées surtout par des massifs de l'étage montagnard de type hêtraie-sapinière relevant du Luzulo- Fagetum, les chênaies en question apparaissent comme des futaies, ou des futaies sur souche de qualité médiocre : les arbres sont tortueux et de faible taille. En ce qui concerne les conditions 3 stationnelles, elles occupent des versants raides, voire escarpés de nature géologique indifférente, avec de nombreux affleurements rocheux. Le sapin, l'épicéa surtout peuvent être présents même au coeur de ces chênaies ; mais ils restent alors cantonnés en sous-étage, ce qui peut être interprété soit comme le signe d'une dynamique progressive sous le couvert de la chênaie, soit comme le résultat de l'action des forestiers (ce qui est évidemment fort peu probable), soit plutôt comme un indice d'une inadéquation stationnelle, les conditions permettant l'installation de quelques semis, mais non leur maintien sur une longue durée et leur intégration à la strate dominante. L'année 2003, marquée par une sécheresse exceptionnelle, a été fatale à un très grand nombre de ces jeunes résineux, ce qui semble appuyer l'hypothèse d'une inadéquation stationnelle de ces versants séchards pour les résineux, en tout cas en l'absence d'une intervention énergique des forestiers. L'existence de la chênaie peut donc sans doute être relativement stable et durable, du moins dans les conditions actuelles.

Dans certains cas, il est vrai, les peuplements de chêne et en particulier certains des plus réduits en surface semblent plutôt résulter d'une dynamique de recolonisation. C'est le cas pour les chênaies claires, assez diversifiées, mêlées par exemple de genévriers, de sorbiers, et de quelques résineux, qui se sont installées sur d'anciens " pâtis » ou " pâquis ", ces pâturages si étendus autrefois dans les paysages d'infieldoutfield ${ }^{4}$ des Hautes Vosges. L'examen des sources cadastrales anciennes et récentes, ou même l'observation des murets qui délimitaient ces pâturages permet alors d'éclaircir le problème ; dans ces conditions, le chêne apparaît comme une essence post-pionnière qui, après un premier stade d'enfrichement, annonce l'installation pérenne d'une végétation forestière relativement stablilisée, au sein de laquelle il pourrait n'occuper qu'une place réduite. Cependant, d'autres chênaies occupent des stations qui sont restées forestières dans les derniers siècles, des "vieilles forêts " selon la distinction établie par Paul Arnould (Arnould 1991). Elles sont alors situées en forêt communale ou domaniale. Parmi les sites concernés, l'essentiel se trouve aujourd'hui dans les Hautes Vosges, sur les versants d'endroit (ou d'adret) dominant Rochesson, Sapois, et Vagney (photo 1). Quelques peuplements d'étendue très limitée se retrouvent à proximité de 
Pétempré à l'amont de Granges sur Vologne, et de très rares petites chênaies se distinguent dans les Vosges gréseuses, comme au col de la Chapelotte.

Photo 1 : Chênaie montagnarde au Droit de Rochesson (Vosges)

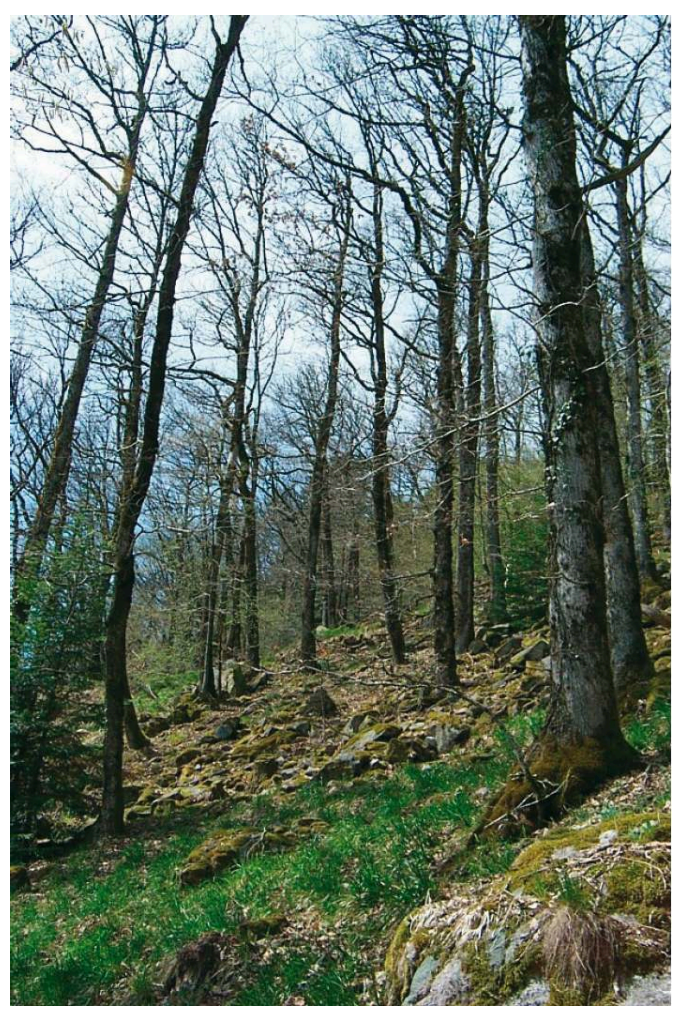

Ainsi, la chênaie (communale) du bois du Senet à Sapois (hautes Vosges) s'étend à une altitude de 550 à $740 \mathrm{~m}$ environ, sur un versant xérique escarpé dominant la vallée de Ménaurupt dans sa section aval. Sur granites de Remiremont et microgranites, elle occupe une pente riche en blocs et en affleurements, au sol maigre, exposée au SudSud-Est. Sur le sommet, un muret ancien à pierres sèches (sans doute lié aux anciennes pratiques de pâturage en forêt) matérialise la ligne de crête sur un replat sommital peu marqué, où domine une formation d'allure très anthropique dominée par l'épicéa en peuplement serré, composée d'arbres mal conformés au houppier court. Le sous-bois est évidemment très pauvre : on note surtout quelques taches de Festuca gigantea. Dès le haut du versant, apparaît le chêne, mêlé d'abord de quelques rares sapins (Abies alba) et pins sylvestres (Pinus sylvestris) ; au coeur du peuplement, les résineux disparaissent tout à fait. Seuls quelques hêtres et houx accompagnent le chêne sessile dans ce qui apparaît comme une médiocre futaie de chênes peu élevés et de mauvaise conformation. En sous-bois apparaît Deschampsia flexuosa avec quelques Dryopteris. A l'aval, le peuplement se mêle à nouveau d'épicéas et de sapins. L'ensemble couvre une surface d'une quarantaine d'hectares si l'on ne tient pas compte des chênaies voisines du même versant, séparées par des peuplements résineux.

Ces modestes chênaies posent un problème non négligeable en termes d'histoire de l'environnement. En effet, couvrant dans leur totalité quelques centaines d'hectares seulement, elles ne peuvent suffire à expliquer l'abondance, voire l'omniprésence des chênaies dans les archives de l'Epoque moderne, et doivent donc être considérées comme des vestiges, ou des faciès relictuels. 


\section{B. Des faciès pourtant omniprésents en archives}

11 En effet, la chênaie apparaît constamment à la lecture des descriptions laissées par les forestiers des XVIe au XIXe siècle. Ainsi, l'une des sources écrites les plus anciennes pour la connaissance des forêts vosgiennes, la "visite " (description) des forêts du comté de Salm en date de 1577, mentionne dans le Val de Senones des forêts «peuplées en leurs pendans de bois de chesne » (AD.M.M. H 1411) alors que le Val de Senones (pris dans sa définition historique) est aujourd'hui exempt de chênaies. En 1719, la visite des forêts de la Châtellenie de Rambervillers mentionne des versants exposés au Sud, souvent médiocrement peuplés de "vieux chesnes deffectueux et rabougris » sur des versants aujourd'hui occupés par le pin, voire par la hêtraie-sapinière (ADV E dpt 374 DD 17). En forêt d'Etival, le procès-verbal de visite rédigé par le grand forestier Florent Bazelaire de Lesseux et ses adjoints en 1741 mentionne « un chenage rabougri, et mal tourné, sur fond de roche », parfois mêlé de pins ou " pinasses »: « les pentes et les crouppes de ces coteaux a l'aspect du midy, sont chargés de bruyères, et d'un mauvais chenage, rabougri, noüeux et mal tourné, sur un fond sec et aride de gros gravier melé de pierraille qui brule a cet aspect, echaude le germe du sapin qui n'y croit point, et ne donne pas assez de nourriture au chêne pour y bien croître » (ADV 17H 52).

Ces « mauvais chenages ", toujours en versant sud, sont généralement désignés comme étant des " rapailles ", terme ambigü qui désigne selon les cas des réalités diverses : tantôt de mauvaises forêts, tantôt des forêts communales que l'on divise en " hautes rapailles " (hêtraies-sapinières exploitées pour le bois d'oeuvre) et " basses rapailles " (taillis ou taillis sous futaie feuillus, normalement dominés par le chêne). Dans le cas des chênaies décrites, de statut domanial (forêts appartenant au seigneur haut justicier mais grevées de droits d'usages), le mot rapaille souligne évidemment la mauvaise qualité de ces forêts.

13 Ces descriptions peu précises ne concernent que les Vosges gréseuses. Les forêts des Hautes Vosges étant gérées de manière bien moins intensive, en raison de l'absence d'enjeux industriels ou financiers qui pourraient justifier une forte activité des officiers ducaux, les descriptions sont vagues et généralement de peu d'intérêt : des faciès communs aujourd'hui comme la hêtraie sommitale, l'érablaie d'éboulis ou les chênaies de versant sud n'y apparaissent pas, ou seulement à travers de rapides allusions.

Les mentions de chênaies montagnardes sont cependant suffisamment nombreuses, et concernent assez souvent des versants aujourd'hui occupés par la hêtraie-sapinière, ou par des faciès anthropisés, pour qu'on s'interroge sur l'étendue réelle, la valeur, la fonction de ces chênaies avant leur quasi-disparition sur ce versant des Vosges. Si les descriptions et autres sources textuelles sont insatisfaisantes, car trop allusives, les archives offrent heureusement des informations plus précises, quantifiées et bien localisées, sous la forme de registres de martelages.

\section{Sources d'archives disponibles : l'intérêt des registres de martelages}

15 Dans le domaine forestier, la recherche en archives est presque totalement dépendante des archives administratives et judiciaires, et son efficacité est donc conditionnée par 
l'existence et l'activité des grueries et maitrises des Eaux et Forêts qui fonctionnèrent jusqu'en 1791.

L'administration lorraine des Eaux et Forêts a suivi, dans son évolution, une chronologie relativement similaire à celle du puissant voisin français. Apparue au Moyen Âge, répartie en grueries gérées par des officiers spécialisés (les gruyers) dès le XIVe siècle (Guyot 1889), elle fut d'abord le bras armé de la politique des ducs sur leurs propres forêts, puis, en vertu de l'importance stratégique des ressources en bois, sur l'ensemble des espaces boisés du duché. La politique ducale eut pour obsession de généraliser le traitement en taillis sous futaie dans les forêts feuillues, tout en ayant soin d'autoriser le maintien de traitements mieux adaptés en forêt résineuse (contrairement à ce que fit le voisin français). Cette administration dotée également de pouvoirs judiciaires eut d'abord pour base juridique une législation cumulative et complexe, constituée petit à petit du XIVe au XVIIe siècle, avant de pouvoir s'appuyer sur un Code cohérent, imité de l'Ordonnance colbertienne de 1669, et promulgué en 1701. Cette législation spécifiquement lorraine resta en place pendant près d'un siècle, y compris après le rattachement au royaume de France en 1766, jusqu'au nivellement révolutionnaire.

Les archives des forestiers deviennent vraiment abondantes à partir du XVIe siècle, lorsqu'une pénurie relative accrut la valeur du bois et des espaces boisés. Dès lors, l'utilisation des archives semble souhaitable, voire indispensable dans toute étude sur le passé des espaces boisés dans les quatre à cinq derniers siècles. Il est vrai que la plupart des documents disponibles apportent peu : descriptions vagues et trompeuses, mâtinées de considérations esthétiques ; requêtes et correspondances biaisées où les intérêts du rédacteur, marchand de bois ou syndic de communauté, masquent probablement la réalité ; plans maladroits où le décor l'emporte sur l'information. Mais d'autres types d'archives sont d'un tout autre intérêt. A partir de la fin du XVIIe siècle, les forestiers lorrains enregistrent toutes leurs opérations de terrain dans des registres spéciaux appelés registres de martelages ou registres d'assiettes. Chaque arbre marqué est donc systématiquement relevé par écrit ; les officiers forestiers prennent soin de spécifier son essence, sa classe d'âge ou de diamètre, sa localisation, son rôle : baliveau, pied-cornier, tournant, rentrant, arbre de lisière. Dans le cas des exploitations en taillis ou en taillis sous futaie, un arpenteur complète les informations apportées par un croquis coté en toises, qui peut permettre de localiser chaque coupe avec une précision satisfaisante, au sein des massifs dont on peut ainsi connaître la surface et la forme même en l'absence de plans forestiers.

Ce corpus impressionnant a parfois été conservé. Les registres des grueries et maîtrises de Nancy, Pont-à- Mousson, Saint-Dié, ou Dieuze, par exemple, renferment des données en quantité pléthorique concernant essentiellement la période 1697-1791. Ainsi peuton, à partir d'une étude par sondages, reconstituer les traits essentiels des forêts lorraines au siècle des Lumières. Les registres étudiés jusqu'ici dans le contexte vosgien sont ceux de la maîtrise de Saint-Dié, rassemblés aux Archives Départementales des Vosges sous les cotes B 525 [A à C] et B 4657 (photo 2). Ils concernent une surface suffisante pour prendre en compte la totalité de l'étagement des Vosges lorraines : hêtraies-chênaies collinéennes, hêtraies, hêtraies-sapinières et hêtraiessapinièrespessières montagnardes. 
Photo 2 : Croquis d'une coupe, extrait d'un registre de martelages de la maîtrise de Saint-Dié (Archives Départementales des Vosges, B 525)

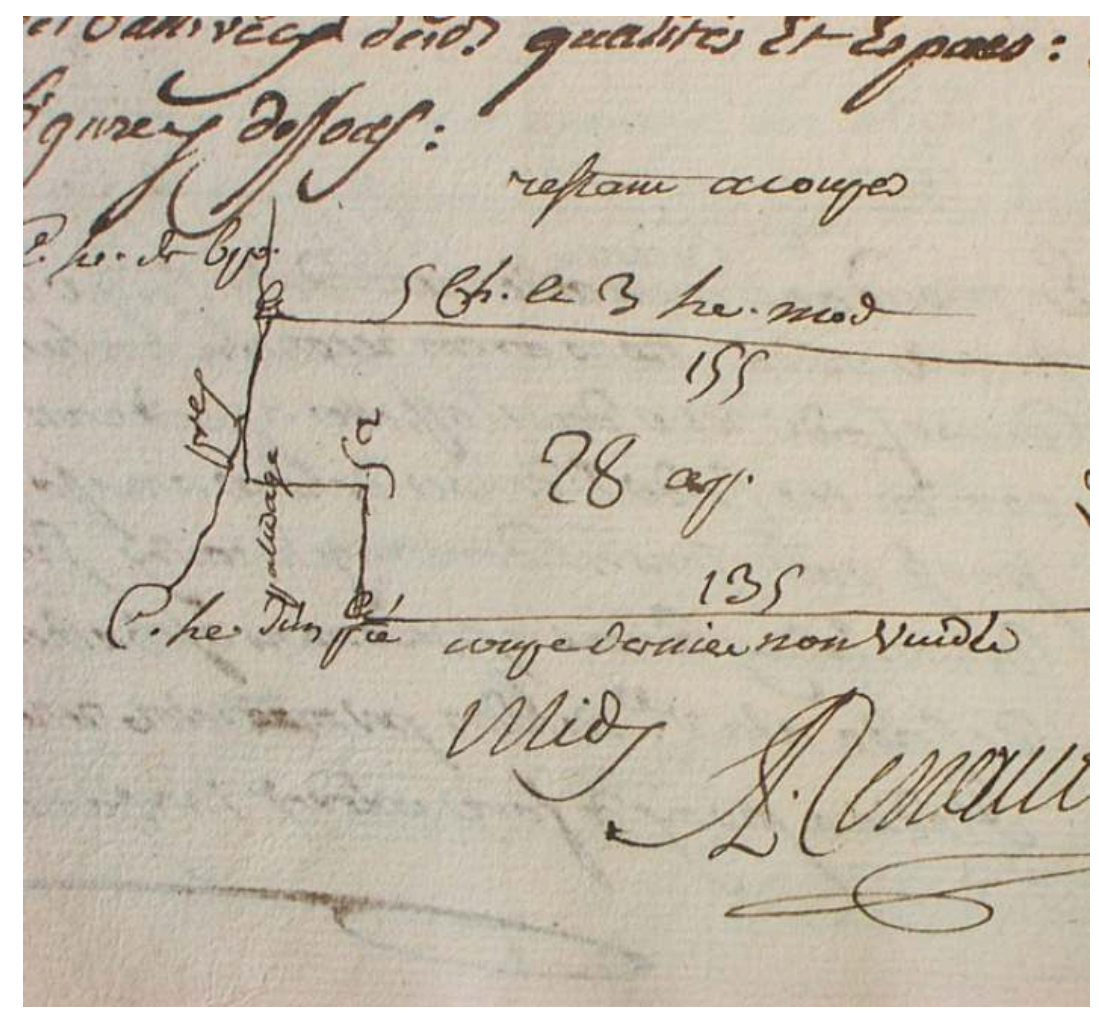

19 A l'Ouest, un pâturage et un pré au long d'un ruisseau ; au Nord, le « restant à couper " (forêt non exploitée) séparé par 8 arbres parois dont 5 chênes et 3 hêtres ; à l'Est, une coupe âgée de 4 à 5 ans; au Sud, la coupe de l'an précédent, encore en exploitation. Les cotes sont en toises de Lorraine $(2,86 \mathrm{~m})$.

L'intérêt premier d'une telle étude relève de l'histoire de la sylviculture. Les forêts lorraines connaissent alors l'apogée du modèle sylvicole lorrain, assez différent du modèle français issu de la législation colbertienne des années 1660. Ces considérations ne seront pas ici prises en compte. Mais outre l'histoire de la sylviculture, la géohistoire et l'histoire de l'environnement peuvent elles aussi trouver dans les registres de martelages les données objectives et quantifiées qui leur manquent trop souvent. Il est ainsi possible de connaître les surfaces forestières alors gérées par les officiers ducaux, l'aspect des lisières, l'habitat riverain composé de « huttes » et « baraques ». Surtout, la composition des forêts lorraines peut être approchée à l'aide de données chiffrées et fiables, de même que la structure des peuplements en taillis sous futaie qui peut être étudiée à travers la densité de réserves des quatre classes d'âge utilisées par les forestiers lorrains : baliveaux de l'âge du taillis (qui seront appelés plus simplement baliveaux), modernes, anciens, et vieilles écorces. De plus, les croquis réalisés, lors de chaque martelage, par l'arpenteur de la maitrise autorisent des essais de cartographie. Ces croquis sont cotés en toises ou verges (la toise de Lorraine valant 2,859 m) ; les coupes se suivant en tire et aire, il est possible de retracer un par un ces croquis sur un fond de carte récent, jusqu'à obtenir le plan de l'ensemble des coupes délimitées par les forestiers du temps des Lumières, un peu à l'image d'un puzzle, ou d'une photographie dont le temps de pose serait de 25 à 30 ans. 


\section{Résultats : les rapailles de chêne au XVIIle siècle}

\section{A. Place des chênaies dans le corpus étudié}

21 L'échantillon étudié à l'échelle de la maîtrise représente au total 1783 assiettes de coupe sur 5935 hectares, avec 286668 arbres relevés avec précision (essence, classe d'âge) : il s'agit généralement soit d'arbres faisant fonction de bornes délimitant la coupe (piedscorniers, parois, tournants et rentrants) soit d'arbres réservés dans la futaie (baliveaux, modernes, anciens, vieilles écorces). Sur ce total, on retiendra les coupes dominées par le chêne, et concernant ce que l'on considère aujourd'hui comme relevant de l'étage montagnard, les limites retenues étant établies à partir de la carte de la végétation du CNRS. Remarquons que les seules coupes de taillis simples observées dans ce corpus ne concernent que le versant alsacien ${ }^{5}$, et n'ont donc pas été prises en compte; le traitement en taillis sous futaie semble absolument généralisé sur le versant vosgien, en tout cas dans les forêts du ressort de la maîtrise de Saint-Dié.

Les principales chênaies encore observables de nos jours ne sont malheureusement pas incluses dans ce corpus : elles se trouvent toutes sur le territoire de l'ancienne maitrise d'Epinal, dont les registres de martelages n'ont été conservés que de façon très partielle et ne permettent pas une étude rétrospective sérieuse. En outre, les quelques modestes chênaies qui, aujourd'hui, existent sur le territoire de l'ancienne maîtrise de Saint- Dié n'apparaissent pas non plus dans les archives étudiées. Une étude comparative à plus de deux siècles d'intervalle n'est donc pas envisageable. Néanmoins, les chênaies montagnardes sont très présentes dans les registres étudiés. Sur les 1783 assiettes étudiées, 486 concernent la montagne et sont donc susceptibles d'être évoquées ici. Si l'on écarte 31 coupes en hêtraie, il reste 455 assiettes de taillis sous futaie en montagne, sur un total de 1568 hectares, qui sont dominées par le chêne. Toutes ces coupes portent sur des peuplements relativement homogènes et nous montrent clairement l'aspect des chênaies montagnardes entre 1747 et 1791.

\section{B. Des taillis pauvres?}

Les informations apportées concernent le peuplement après exploitation ; l'état du taillis n'est donc pas connu, si ce n'est par de très brèves descriptions. La révolution n'est pas fixée mais, lorsqu'elle a pu être établie, évolue généralement entre 20 et 40 ans, ce qui est long pour le XVIIIe siècle. Au bois du Recreux (basses Vosges gréseuses), la cartographie des coupes a montré leur retour sur le même point au bout de 40 ans ; en forêt de Badonviller (massif du Donon), après 19 ans ; dans les rapailles de la Bure dominant Saint- Dié, 20 ans.

La place des résineux, lorsqu'ils sont présents, ne pouvant être quantifiée ${ }^{6}$, on ne retient que les coupes, très majoritaires, dans lesquelles ni le pin, ni le sapin ne sont mentionnés. Le chêne représente au total 86,6 \% des arbres marqués comme limites de coupe par les forestiers, et 90,1\% des arbres marqués comme baliveaux, modernes, anciens ou vieilles écorces. Le hêtre, le bouleau, le tremble se partagent le reste. Il est vrai que cette domination sans partage résulte d'un choix; le peuplement avant exploitation a pu contenir, dans des proportions inconnues, d'autres essences que les forestiers n'ont pas jugées dignes d'être épargnées. Mais cette sélection elle-même 
reste une information fondamentale, tant la coupe répétée des essences secondaires avant leur maturité a pu fortement et durablement marquer les forêts en question.

La qualité de ces chênaies est peu appréciée, si l'on en croit les descriptions annexées aux procès-verbaux de coupes. Les peuplements sont " de mauvaise crute ", " chargés de broussailles et bruyères ", poussant sur " un sol sec et aride " ou encore " sec et pierreux »; les arbres sont de " mauvais chenages mêlés de bois blancs » (c'est-à-dire d'essences sans valeur, bouleau, tremble ou sorbier) ou, pire, de « pinasses » que l'on abandonne volontiers à l'exploitation avec le taillis

Mais ces informations peuvent être précisées à la lueur des informations sur le balivage. En ce qui concerne la structure de ces forêts, l'échantillon est loin d'être aussi important qu'on pourrait le souhaiter ; en effet, dans une majorité de cas, les chênaies montagnardes sont composées de jeunes arbres rabougris « qui ne peuvent souffrir l'empreinte du marteau ", ou de broussailles impénétrables ; les forestiers se contentent donc de demander le respect d'un nombre variable de baliveaux par arpent, se réservant de vérifier la qualité de l'exploitation lors du récolement ${ }^{7}$; on ne connaît donc pas le nombre exact des arbres réservés dans les différentes classes d'âge.

Les coupes dont on peut connaitre la structure se caractérisent par une assez forte densité de baliveaux de l'âge (balivage dit « dense ») avec une moyenne de 52 tiges par hectare ; les modernes sont encore présents en densité satisfaisante (28 par hectare, ce qui correspond grossièrement à la moyenne de la maitrise dans son ensemble); mais les classes d'âge supérieures apparaissent très déficitaires, avec 7 anciens et 4 vieilles écorces par hectare seulement. La difficulté d'établir un balivage digne de ce nom est parfois sensible pour les baliveaux de l'âge eux-mêmes. Dans le cas d'une coupe délivrée à la communauté des Cours en forêt de Hennefête ${ }^{8}$, en 1752, les officiers déodatiens vont jusqu'à baliver 31 "boules » (bouleaux) et deux trembles, essences normalement inadaptées pour une intégration dans la réserve, mais dont l'apport était nécessaire pour tenter de respecter numériquement les prescriptions des ordonnances.

La structure observée est donc très pauvre en gros bois lorsqu'on la compare avec les autres peuplements de la maîtrise, surtout dans les hêtraies où la richesse en anciens et vieilles écorces est telle que l'on constate parfois des pyramides des âges inversées, avec des arbres âgés plus nombreux que les plus jeunes, comme si une futaie vieillie et régularisée dans les gros bois et les bois moyens entravait la régénération et plus généralement le fonctionnement du taillis sous futaie (Rochel 2004, 2008). La richesse du balivage est loin d'atteindre les quantités prescrites, par exemple, par l'arrêt de 1765 censé la réglementer. Mais le tableau 1 montre que le balivage des chênaies reste globalement dans la norme observée au XVIIIe siècle. Le discours des forestiers, toujours prompts à décrier la pauvreté des rapailles, peut donc être relativisé à condition de garder en mémoire le fait que le balivage n'est connu que pour une minorité de coupes, évidemment les plus riches, dans lesquelles le balivage avant exploitation a été jugé possible par les forestiers déodatiens. 
Tableau 1 : Structure moyenne du balivage dans la maîtrise de Saint-Dié et éléments de comparaison

\begin{tabular}{|l|c|c|c|c|c|}
\hline & Source & Baliveaux & Modernes & Anciens & Vieilles écorces \\
\hline Maîtrise de Saint-Dié & A.D.V. B 525 & 41 & 30 & 18 & 7 \\
Chênaies montagnardes & A.D.V. B 525 & 52 & 28 & 7 & 4 \\
Moyenne en Bourgogne, 1730 & Corvol, 1984 & 48 & 19 & \multicolumn{2}{|c|}{ Réserves de droit } \\
Ordonnance de 1669, France & - & 31 & Réserves de droit & Réserves de droit & 11 \\
Arrêt du 2 mars 1765, Lorraine & - & 58 & 19 & 19 & 10 \\
\hline
\end{tabular}

\section{Des chênaies d'adret}

La cartographie des coupes, lorsqu'elle s'est révélée possible, confirme l'observation actuelle de peuplements presque exclusivement localisés en versant Sud, et sur des pentes fortes ; il en est ainsi dans les bois du Recreux et de Charmelot qui ont pu être partiellement reconstitués à partir des informations apportées par les registres de martelages.

Figure 1 : Mentions de rapailles de chêne sur le territoire de la maîtrise de Saint-Dié (1747-1791)

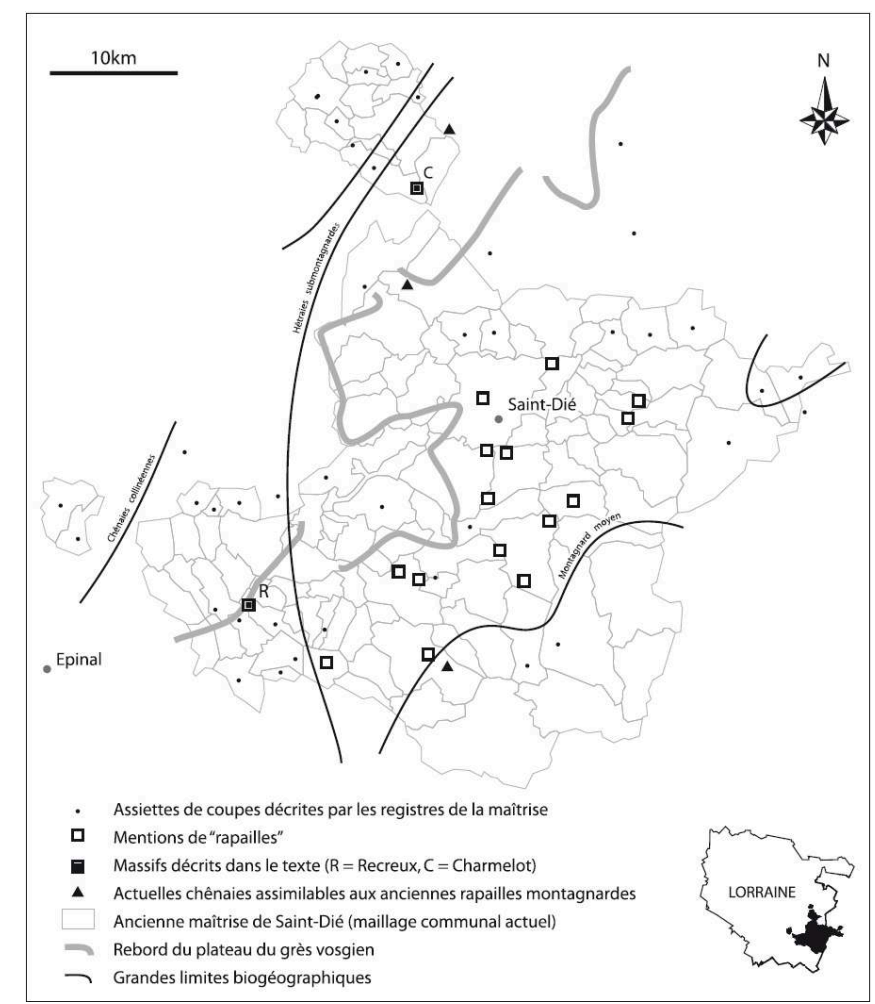

Le bois du Recreux, situé à la limite occidentale de la maitrise de Saint-Dié, est très caractéristique de l'étage montagnard inférieur, très anthropisé, en ce qu'il est aujourd'hui largement enrésiné en pins et sapins principalement, et secondairement en douglas et en épicéa. Occupant 195 hectares au total, il est situé sur le plateau du grès vosgien et son versant sud, surplombant la large vallée de la Vologne, à une altitude modeste (450 à 587 mètres). L'étape cartographique du travail imposait de localiser et cartographier les coupes successives ; le travail ne s'est pas révélé aussi simple que prévu. De 1749 à 1759, le résultat obtenu a été trop approximatif pour être jugé digne d'intérêt. L'étude est donc limitée aux coupes de 1760 à 1770 et de 1773 à 1790, ces deux 
séries étant interrompues par une lacune de deux années. Les croquis des arpenteurs successifs se sont révélés assez approximatifs ; les angles ne sont jamais précisés, ce qui gêne la restitution de la forme exacte des assiettes. Néanmoins, une reconstitution satisfaisante de la marche des coupes a été obtenue ; elle couvre les trois quarts de la surface $d u$ bois. L'analyse révèle une localisation sans surprise des deux faciès. Les peuplements dominés par le hêtre occupent principalement la surface du plateau, ainsi qu'une basse abritée au Sud-Est du massif ; sur ces stations favorables, la hêtraie est riche en gros bois, voire régularisée au Nord. Les coupes où la part du chêne est supérieure à la moyenne sont situés sur les versants plus xérophiles, exposés au Sud ou au Sud- Ouest ; il s'agit d'une rapaille de chêne, pauvre en réserves, marquée par la présence importante du bouleau (figure 2). Cette rapaille a disparu depuis. La chênaie ne représente plus aujourd'hui qu'un bouquet mêlé de pins en bordure de plateau ; le reste été enrésiné en pins et en douglas.

Figure 2 : Reconstitution des coupes et des peuplements après exploitation au bois du Recreux (Commune de Deycimont, basses Vosges)

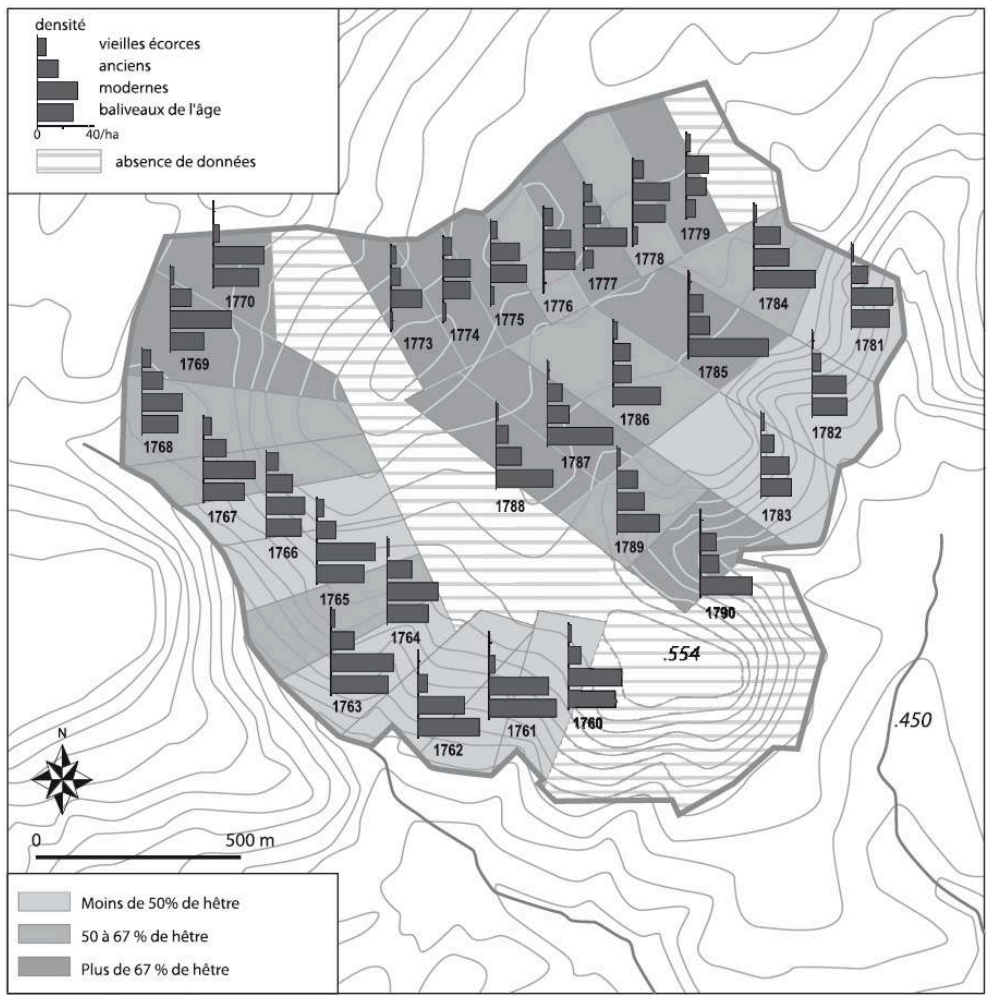

Le haut de Charmelot, partie de l'actuelle forêt domaniale des Elieux, a pu, lui aussi, faire l'objet d'un essai de cartographie à partir des procès-verbaux des coupes de 1755 à 1765 (figure 3). Les peuplements, aujourd'hui en partie ennoyés par le lac du Vieux Pré à Pierre- Percée, sont majoritairement feuillus, mêlés de sapins en faibles proportions, systématiquement réservés par les forestiers. On remarque l'opposition très claire entre deux faciès qui apparaissent à la fois dans les descriptions données par les forestiers, dans le pourcentage de chênes, et dans la structure de la réserve. En versant frais, le hêtre domine, mêlé de sapins ; la réserve peut atteindre les densités prescrites par le règlement de 1701. En versant exposé au sud, le peuplement est pauvre, la réserve est très déficiente et le chêne mêlé de quelques pins et sapins l'emporte largement sur le hêtre : il s'agit clairement d'une rapaille montagnarde. Le chêne est 
aujourd'hui absent des sapinières et pineraies qui occupent le haut (non ennoyé) des versants.

Figure 3 : Reconstitution des coupes et des peuplements après exploitation au haut de Charmelot (Commune de Pexonne, massif du Donon)

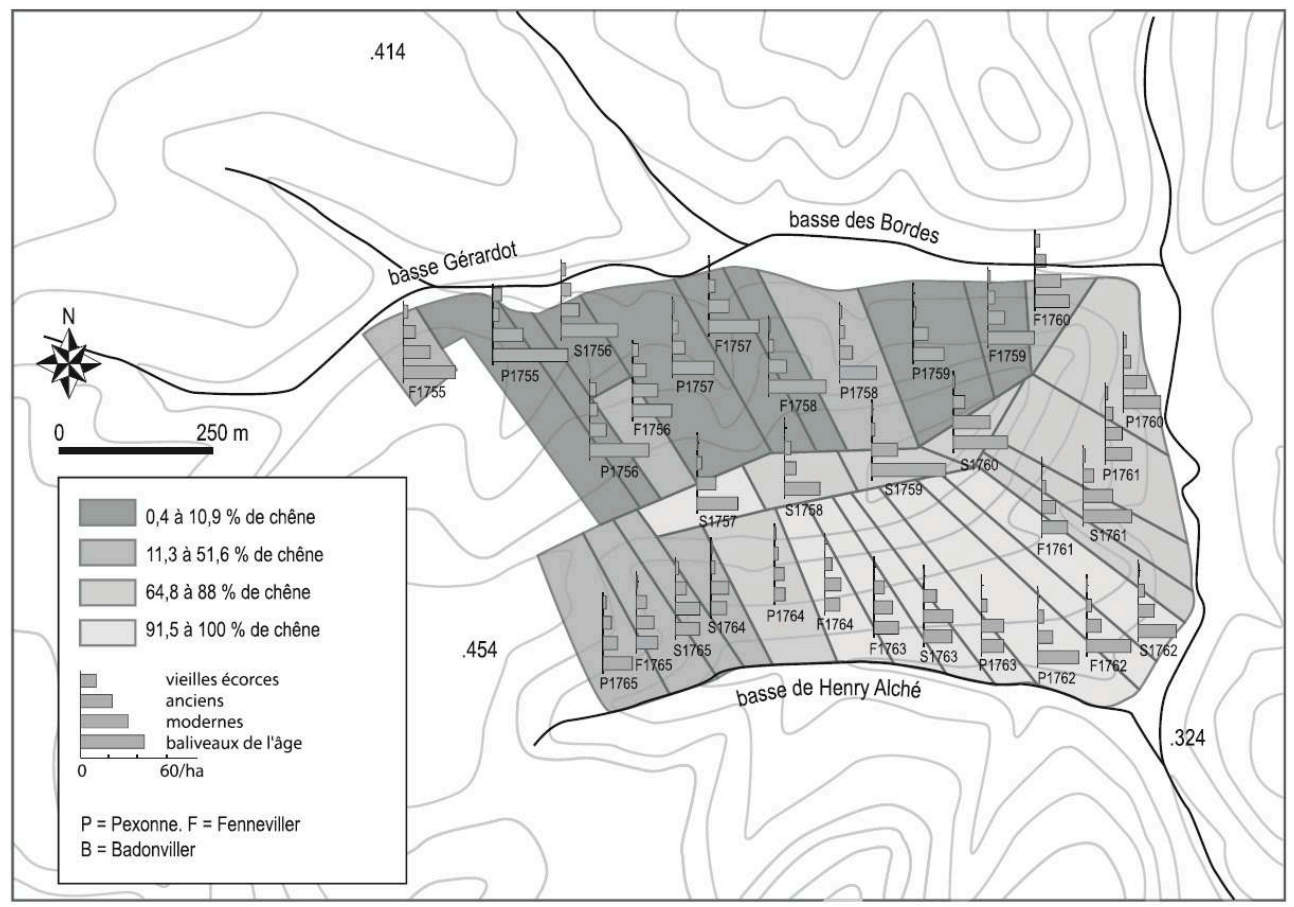

\section{Conclusion}

Les rapailles montagnardes occupent des surfaces considérables dans la montagne vosgienne du XVIIIe siècle, presque toujours en situation d'adret. Il serait vain, à partir des informations disponibles, d'essayer d'en mesurer la surface. Il s'agit toujours de taillis sous futaie bien plus pauvres que le sont généralement les forêts lorraines à la même époque. Cette structure pauvre en réserves pourrait être interprétée de deux façons. Il pourrait s'agir d'un héritage : le traitement en taillis sous futaie serait récent, et succèderait à un taillis simple, d'où le manque d'arbres de valeur. Cette explication est peut-être valable pour certains massifs ; mais les réserves âgées ne sont pas plus abondantes à la fin de la période étudiée, après théoriquement au moins un passage. La quasi-absence de baliveaux anciens ou de vieilles écorces s'explique donc davantage par la médiocrité des stations en cause, sans doute appauvries par plusieurs siècles d'exploitation intensive : les chênaies en question, presque toujours en situation d'adret, sont situées à proximité immédiate de villages ou hameaux et cette situation vaut à la fois pour les chênaies documentées par les archives du XVIIIe siècle et pour celles encore observables de nos jours.

Rien ne doit surprendre dans la disparition des chênaies qui figurèrent parmi les premières cibles du zèle des reboiseurs. Si les Vosgiens interrogés sur leurs pratiques forestières lors de l'enquête de 1783 évoquent l'importance des «basses rapailles » dans leur approvisionnement en bois de feu, les forestiers considèrent alors déjà le chêne comme un intrus en montagne, et la chênaie comme un faciès de faible valeur. D'une 
manière générale, les résineux apportent des revenus confortables aux propriétaires de forêts, tandis que les feuillus sont bien moins recherchés, au point qu'au début du XIXe siècle, dans la montagne vosgienne, la valeur d'une forêt feuillue est deux à trois fois moins élevée qu'une sapinière de même surface (Rochel 2004).

Les archives n'apportent ici que des informations ponctuelles, lacunaires, dont la continuité avec l'actuel est problématique en raison de la rapidité des enrésinements dès la première moitié du XIXe siècle, période pauvre en archives. Le caractère anthropique ou non des chênaies doit être recherché dans des périodes plus reculées. Les connaissances encore peu satisfaisantes sur la chênaie seraient grandement améliorées par une étude de type anthracologique / pédoanthracologique sur les sites documentés en archives, tels que le bois du Recreux ou la forêt de Mortagne.

\section{BIBLIOGRAPHIE}

AGNOLETTI M. ANDERSON S. (Ed.) 2000. - Methods and Approaches in Forest History. CABI publishing, IUFRO Series $\mathrm{n}^{\circ} 3,281 \mathrm{p}$.

ARNOULD P. (1991). - Forêt, nouvelles forêts, vieilles forêts. Dans Corvol Andrée (Ed. ) La Forêt, actes du 73e congrès national des sociétés savantes. Paris : CTHS, p. 13-30.

BONHÔTE J. (1998). - Forges et forêts dans les Pyrénées ariégeoises. Aspet, PyréGraph, 337 p.

CORVOL A. (1984)._L'Homme et l'arbre sous l'Ancien Régime. Paris : Economica, 1984, 757 p.

DION J. (1985). - Les forêts vosgiennes. Etude biogéographique. Paris : aux Amateurs de Livres, $484 \mathrm{p}$.

DUBOIS J.-J. (1986). - L'utilisation des archives forestières et l'étude rétrospective des peuplements : un essai de cartographie statistique dans la forêt du Nouvion (Thiérache) au XVIIIe siècle. Hommes et Terres du Nord, 2-3 1986, p. 214- 220.

DUBOIS J.-J. (1989). — Espaces et milieux forestiers dans le Nord de la France. Etude de biogéographie historique. Thèse de Doctorat d'Etat, Univ. Paris I, 2 t. 1023 p.

DUPOUEY J.-L., DAMBRINE E., DARDIGNAC C., GEORGES-LEROY M. (Textes réunis par) (2007). - La mémoire des forêts. Actes du colloque "forêt, archéologie et environnement ", Nancy, 14-16 décembre 2004. ONF, DRAC, INRA-Lorraine, s.1. 2007, 294 p.

GARNIER E. (2004). - Terre de conquêtes. La forêt vosgienne sous l'Ancien Régime. Paris : Fayard, $620 \mathrm{p}$.

HOUZARD G. (1980). - Les massifs forestiers de Basse- Normandie : Brix, Andaines et Ecouves. Essai de biogéographie. Thèse de Doctorat d'Etat, géographie, Université de Caen. 2 vol., 667 p.

HUSSON J.-P. (1991). - Les hommes et la forêt en Lorraine. Paris, Bonneton, 318 p.

JACAMON M. (1983). - Arbres et forêts de Lorraine. Colmar : S.A.E.P. 141 p.

JACAMON M., TIMBAL J. (1975). - Carte de la végétation, feuille nº 27 : Nancy. CNRS. 
MONCHEREL (1778). - Commentaire sur les ordonnances de Lorraine, civile, criminelle, et concernant les eaux et forêts, combinées avec celles de France. Bouillon : Société Typographique, pagination multiple.

NÖLKEN W. (2005).- Holzkohleanalytische Untersuchungen zur Waldgeschichte der Vogesen. Thèse, biologie, Université Albert-Ludwig de Fribourg, 182 p.

ROCHEL X. (2004a). - Gestion forestière et paysages dans les Vosges d'après les registres de martelages du XVIIIe siècle. Essai de biogéographie historique. Thèse, géographie, université Nancy 2, 2004, 489 p.

ROCHEL X. (2004b). - In search of vanished broad-leaved forests in the Vosges mountains. Colloque « Woodlands : Cultural Heritage », I.U.F.R.O. 3-5 mai 2004, Mariabrunn, Vienne (Autriche). Publié dans : internationale IUFRO Konferenz Kulturerbe Wald. News of Forest History, III, 1-2005, p. 85-90.

ROCHEL X. (2007). - « Comment reconstituer les forêts du XVIIIe siècle ? L'exemple des hêtraieschênaies lorraines. » Colloque Géoflore 2007, Cartographie de la flore, de la végétation et des milieux, Nancy, 10-12 mai 2007. Publié dans : Actes du colloque Géoflore 2007. L.A. SE.R. / Bulletin de l'association des botanistes lorrains, 1-2007, p. 140-149.

ROCHEL X. (2008). - « Sources d'archives et histoire de l'environnement : l'origine des hêtraies vosgiennes d'après les registres de martelages du XVIIIe siècle ». Colloque de Chilhac, 27-30 septembre 2006. Dans GALOP Didier (Dir.) Paysages et environnement. De la reconstitution du passé aux modèles prospectifs. Besançon : Presses Universitaires de Franche-Comté, 2008, p. 77-86.

\section{NOTES}

1. On exclura ici les Vosges du Nord, au-delà du seuil de Saverne, où les réalités biogéographiques et géohistoriques sont très différentes du massif vosgien proprement dit (Jéhin 2003).

2. Dans le domaine forestier, le mot « peuplement " ne répond pas à sa définition la plus courante en géographie, et désigne simplement un ensemble d'arbres.

3. Les données statistiques présentées sont celles correspondant au dernier cycle d'inventaire de l'Inventaire Forestier National pour les territoires concernés.

4. Un système agraire d'infield-outfield juxtapose des surfaces intensément cultivées (infield), de taille réduite, dont la fertilité est conservée à l'aide de transferts (amendements surtout) en provenance de l'outfield, sorte de saltus pâturé extensivement et occupant la majorité de l'espace communal. Le terme d'infield-outfield ne se justifie pleinement que pour certaines localités du coeur des Hautes Vosges.

5. Le duché de Lorraine s'étendait assez loin à l'Est de la ligne de crête, jusqu'à Saint-Hippolyte (Haut Rhin).

6. Les essences résineuses font partie des " réserves de droit », systématiquement incluses dans la réserve, et ne sont donc pas martelées par les forestiers.

7. «Si les officiers ne peuvent dans les délivrances d'affouage ou de taillis, procéder à la marque des baillivaux, ils doivent en exprimer dans leurs procès-verbaux les causes \& les motifs (comme s'il y a impossibilité de pénétrer) \& ordonner à ceux qui doivent les exploiter de laisser des baillivaux en suffisance \& au désir de l'ordonnance. Cette disposition est prévue par arrêt du Conseil du 19 Décembre 1760 ; mais au récolement on fera prudemment de marquer les ballivaux »(Moucherel 1778). 
8. Partie de l'actuelle forêt domaniale de Vologne sur la commune de Corcieux, le petit massif de Hennefête, aujourd'hui enrésiné, est intermédiaire entre hautes et basses Vosges.

\section{RÉSUMÉS}

La chênaie n'occupe aujourd'hui que de très faibles surfaces sur les adrets de la montagne vosgienne. Elle est pourtant omniprésente dans les archives du XVIe au XVIIIe siècle, et la rareté des peuplements actuels ne s'explique que par les enrésinements massifs du XIXe siècle. L'étude des archives des forestiers du temps des Lumières, et en particulier des registres de martelages, permet de reconstituer la composition et la structure de ces chênaies avant leur disparition.

Oak stands are very scarce in the Vosges mountains, but they are the remnants of a once very common landscape feature. Their quasi-disappearance is the result of massive conversions to Conifers during the 19th century. This paper deals with these oak woods as they can be traced back in 18 th century archives.

In den Vogesen wachst der Eichenwald heute auf nur begrenzten Flachen der nach Süden ausgerichteten Hangen. Nach den Archiven aus dem 16 bis $18 \mathrm{Jh}$. war er überall und die Seltenheit der heutigen Eichenwalder ist eine Foige der Aufforstungen mit Fichten im $19 \mathrm{Jh}$. Durch die Erforschung der Forstarchive aus der Zeit der Aufklarung, insbesondere der Hammernregister, wurde die Zusammensetzung dieser Eichenwalder vor ihrem Aussterben rekonstruiert.

\section{INDEX}

Mots-clés : archives, chênaie, enrésinements, martelages

Schlüsselwörter : Archiven, Aufforstungen, Eichenwald, Hämmern

Keywords : archives, marking of trees, oak forest, reafforestations

\section{AUTEUR}

\section{XAVIER ROCHEL}

CERPA - Nancy-université - 23, boulevard Albert $1^{\text {er }}-54015$ Nancy Cedex - Xavier.Rochel@univnancy2.fr 